Research Paper

\title{
GABAB receptor inhibits tumor progression and epithelial-mesenchymal transition via the regulation of Hippo/YAP1 pathway in colorectal cancer
}

\author{
Huihui Wang, MD ${ }^{1,2^{*}}$; Hao Zhang, $\mathrm{MD}^{3^{*}}$; Zhirong Sun, MD, PhD ${ }^{1,2^{*}}$; Wankun Chen, MD, PhD ${ }^{3,4}{ }^{\bowtie}$; \\ Changhong Miao, MD, $\mathrm{PhD}^{1,2,3} \bowtie$ \\ 1. Department of Anesthesiology, Fudan University Shanghai Cancer Center. \\ 2. Department of Oncology, Shanghai Medical College, Fudan University, Shanghai 200032, China. \\ 3. Department of Anesthesiology, Zhongshan Hospital, Fudan University; Cancer Center, ZhongShan Hospital, Fudan University; 180\# Feng-Lin Road, \\ Shanghai, 200032, China. \\ 4. Fudan Zhangjiang Institute, Shanghai 201203, China. \\ *These authors contributed equally to this work.
}

$\triangle$ Corresponding authors: Changhong Miao, M.D., Ph.D. Department of Anesthesiology, Fudan University Shanghai Cancer Center, 270\# Dong-An Road, Shanghai, 200032, China; Department of Anesthesiology, Zhongshan Hospital, Fudan University, 180\# Feng-Lin Road, Shanghai, 200032, China. E-mail: miaochangh@163.com; Wankun Chen, M.D., Ph.D.: Department of Anesthesiology, Zhongshan Hospital, Fudan University, 180\# Feng-Lin Road, Shanghai, 200032, China. E-mail: chenwank@163.com.

(1) The author(s). This is an open access article distributed under the terms of the Creative Commons Attribution License (https://creativecommons.org/licenses/by/4.0/). See http://ivyspring.com/terms for full terms and conditions.

Received: 2021.01.13; Accepted: 2021.04.23; Published: 2021.05.10

\begin{abstract}
Gamma-Aminobutyric Acid Type B Receptor (GABABR) plays essential roles in tumor progression. However, the function of GABABR in colorectal cancer (CRC) needs further clarification. As the main part of GABABR, GABABRI expression was identified significantly lower in tumor tissues than those in non-tumor normal tissues and that $C R C$ patients with high GABABRI expression lived longer. Further studies indicated that knockdown of GABABR 1 elevated CRC cell proliferation, migration, and invasion. Furthermore, knockdown of GABABRI activated the expression of the epithelial-mesenchymal transition (EMT)-related proteins $\mathrm{N}$-cadherin and Vimentin, whereas decrease the protein level of E-cadherin. In addition, activation of Hippo/YAPI signaling contributes to the GABABR1 down-regulation promoted proliferation, migration, invasion and EMT in CRC cells. At last, we verified the contribution of Hippo/YAPI signaling in the GABABR 1 down-regulation impaired biological phenotype of colon cancer cells in vivo. In summary, these data indicate that GABABR 1 impairs the migration and invasion of CRC cells by inhibiting EMT and the Hippo/YAPI pathway, suggesting that GABABR1 could be a potential therapeutic target for CRC.
\end{abstract}

Key words: Gamma-Aminobutyric Acid Type B Receptor; proliferation, migration; invasion; epithelialmesenchymal transition; YAP

\section{Introduction}

Colorectal cancer (CRC) is the third most common cancer and the fourth leading cause of cancer death worldwide, accounting for almost 900,000 deaths in 2018 [1, 2]. Surgery has long been established as the primary treatment for CRC. Benefiting from early diagnosis, recent immunotherapy and anti-tumor agents used for treating CRC, the mortality is remarkably reduced; however, the recurrence rates are discouragingly high, and the median overall survival rate for patients remains unsatisfactory. Hence, the core molecular mechanisms underlying CRC progression need further study, and exploring novel therapeutic targets is crucial to improve the survival rates of CRC patients.

Gamma-amino butyric acid (GABA), which acts on ionotropic $\left(\mathrm{GABA}_{\mathrm{A}}\right.$ or $\left.\mathrm{GABA}_{\mathrm{C}}\right)$ and metabotropic $\left(G_{A B A}\right)$ receptors, is one of the most important inhibitors of neurotransmitters [3]. GABA receptor is pentameric ligand-gated chloride channel, represents the target receptors for major general anesthetics and benzodiazepines. GABA ${ }_{C}$, named GABA-A-rho, is typically classified as a subtype of $\mathrm{GABA}_{A}[4]$. GABA 
receptor, a G protein-coupled receptor (GPCR), functions as a constitutive heterodimer composed of the GABABR1 and GABABR2 subunits. Research increasingly suggests that the $G_{A B A}$ receptor (GABABR) is involved in tumor progression [5]. Previous studies showed that the GABABR antagonist CGP had anti-tumor effects in high-grade chondrosarcoma cells and regulated proliferation of the chondrosarcoma cell line OUMS-27 via apoptotic pathways [6]. Another study indicated that the proliferation and migration of ovarian cancer cells were inhibited by down-regulating the mRNA levels of GABAB receptor [7]. In addition, GABBR1 induces secretion of the peptide gastrin from neuroendocrinelike cells that are involved in prostate cancer progression [8]. Recently, studies have also reported that GABABR regulates the proliferation of colorectal cancer cells by the GSK-3 $\beta / \mathrm{NF}-\mathrm{kB}$ signaling pathway [9]. However, the exact function of GABABR in CRC needs further clarification.

The Hippo pathway is a highly conserved master regulator of organ size and tissue regeneration, and it regulates various biological functions, such as proliferation, apoptosis, viability, and differentiation [10]. Dysregulation of the Hippo pathway is associated with multiple pathological disorders, such as a broad range of cancer types, diabetes, and neurodegenerative diseases [11]. Many studies have revealed that aberrant activation of YAP is one of the most important mechanisms accounting for the progression of cancer. Hippo/YAP signaling has been reported to regulate proliferation, migration, and invasion in pancreatic cancer cells [12]. In gastric carcinoma, Hippo/YAP signaling plays a critical role in epithelial-mesenchymal transition (EMT) [13]. Moreover, the phosphorylation of YAP is involved in the regulation of proliferation and apoptosis in hepatocellular carcinoma [14]. According to recent reports, through downregulation of LATS1 activates YAP, and may contribute to the progression of CRC [15]. However, the exact mechanism remains unclear. Multiple studies have shown that GCPRs are one of many upstream regulators of Hippo/YAP pathway $[16,17]$. Also, GPCR-Hippo pathway was considered as a potential target for cancer molecular targeting therapy [18]. As one of GCPRs, GABABR might be closely associated with YAP.

This study aims to evaluate the role of GABABR in CRC progression. In the present study, we analyzed the relationship between GABABR and the prognosis of CRC patients, the experimental work presented here explored the mechanism of GABABR's role in $C R C$, which may provide new avenues for CRC research.

\section{Materials and Methods}

\section{Immunohistochemistry}

This study was approved by the Ethics Committee of Fudan University Shanghai Cancer Center (FUSCC), China. Tumor tissues embedded in paraffin were cut into $3 \mu \mathrm{m}$ sections, followed by deparaffinization and rehydration. Immunohistochemical staining was performed with antibodies to GABABR1 (Abcam, 1:100, Cambridge, UK) and GABABR2 (Abcam, 1:100) at $4{ }^{\circ} \mathrm{C}$ overnight. The sections were incubated with peroxidase-conjugated secondary antibodies for $1 \mathrm{~h}$ at $37{ }^{\circ} \mathrm{C}$ and were mounted in a mounting medium containing glycerol (Beyotime, China). The analysis of positively stained tumor cells was conducted and images were taken under a light microscope.

\section{Cell culture}

RKO, DLD1, Lovo, HCT116, HT29 and SW620 human CRC cell lines were a kind gift from Prof. Changhong Miao (Fudan University Shanghai Cancer Center, China) and were cultured in DMEM (HyClone, Thermo, USA) supplemented with 10\% FBS (Biological Industries, State of Israel); cells were incubated at $37^{\circ} \mathrm{C}$ in a $5 \% \mathrm{CO} 2$ incubator.

\section{Reagents}

Anti-GABABR1 and anti-GABABR2 mAbs were purchased from Abcam (Cambridge, UK). $\mathrm{N}$-cadherin, E-cadherin, Vimentin and Hippo/YAP1 pathway antibodies were purchased from CST (Danvers, MA, USA). An anti-actin mAb, an anti-GAPDH $\mathrm{mAb}$ and an anti-histone $\mathrm{H} 3$ were purchased from Proteintech (Chicago, USA).

\section{Western blot analysis}

Cells were lysed with lysis buffer (Beyotime, China) containing protease inhibitors, PMSF (Beyotime, China). Cellular proteins were separated by $10 \%$ SDS- PAGE and were then transferred onto a Hybond TM-P membrane (GE Healthcare, UK). The membrane was blocked with 5\% skim milk in TBST for $1 \mathrm{~h}$ at room temperature. Then, the membrane was incubated with primary antibodies overnight at $4{ }^{\circ} \mathrm{C}$. Next, the membrane was incubated with peroxidaseconjugated secondary antibodies for $1 \mathrm{~h}$ after washing with TBST three times. The blots were then treated with a chemiluminescent reagent (Merck Millipore, USA) and visualized on X-ray films (MidSci, USA). All results were repeated for three times.

\section{Q-PCR}

Total mRNA was isolated from RKO and Lovo cells using Trizol ${ }^{\circledR}$ (Invitrogen, Carlsbad, CA). Then, mRNA was reverse transcribed into cDNA. Real-time 
reverse transcription polymerase chain reaction (Q-PCR) was performed to detect the expression of $\mathrm{N}$-cadherin, E-cadherin and Vimentin with iTaq ${ }^{\mathrm{TM}}$ SYBR Green Supermix (Bio-Rad, CA) in a Step One Plus $^{\text {TM }}$ Real-Time PCR System (Life Technologies, USA), according to the manufacturer's instructions (For primer sequences, see Supplementary Table 1). All results were repeated for three times.

\section{Wound healing assay}

Cells were inoculated into a 6-well plate and were cultured to complete confluence. After removing the medium, the cell layers were scraped using a $10 \mu \mathrm{l}$ pipette tip. After rinsing out the scraped cells, the remaining cells were incubated with serum-free medium for $24 \mathrm{~h}$ at $37^{\circ} \mathrm{C}$. The distance of wound closure was recorded to measure the degree of wound healing. All results were repeated for three times.

\section{Transwell assay}

Cell invasion assays were performed in a transwell chamber (24-well, $8 \mu \mathrm{m}$ pore size; Corning). RKO and Lovo cells were resuspended in serum-free DMEM medium, and $6 \times 10^{4}$ cells suspended in $200 \mu \mathrm{l}$ were added to the upper chamber membranes. The bottom was coated with $1 \mathrm{mg} / \mathrm{ml}$ BD Matrigel matrix, and $500 \mu \mathrm{l}$ of complete medium was placed into the lower chamber as a chemoattractant. Then, the cells were incubated at $37^{\circ} \mathrm{C}$ in a $5 \% \mathrm{CO}_{2}$ incubator. After $24 \mathrm{~h}$, the non-invaded cells on the surface of the membrane were removed using a cotton swab. The invaded cells on the lower surface were fixed with $4 \%$ paraformaldehyde and stained with $0.5 \%$ crystal violet. Each experiment was performed in triplicate. The number of invaded cells was counted in 4 fields for each transwell, and the data were analyzed using ImageJ software. All results were repeated for three times.

\section{CCK-8 assay}

Cells were inoculated into a 96-well plate with 2000 cells/well. The wells without cells were used as the blank control group. Each group had five wells. Cell counting kit-8 (CCK-8) reagent was added, and then the cells were incubated for 2 hours in the dark. The wavelength of the enzyme marker was assessed at $450 \mathrm{~nm}$, and the OD value was measured. All results were repeated for three times.

\section{Tumor xenografts}

Five-week-old BALB/c nude mice were purchased from SLAC Animal Center (Shanghai, China) and then used for xenograft tumor model. The animal experiments were approved by the Institutional Animal Care and Use Committee of the Fudan University. The control and lentivirus mediated stable GABABR1 knock-downed Lovo cells were subcutaneously injected to nude mice and then tumor volumes were monitored every 3 days. The YAP1-TEAD inhibitor Peptide 17 (2 ug/ $\mathrm{kg})$ were injected by tail vein every 5 days. Tumor volumes were estimated by length and width and calculated as the following formula:

$$
\text { Tumor volume }=\left(\text { length }{ }^{*} \text { width^}{ }^{\wedge}\right) / 2
$$

About one month later, the nude mice were sacrificed and then tumors were excised, pictured, and weighed.

\section{Statistical analysis}

Statistical analysis was performed with SPSS 17.0 (SPSS Inc., Chicago, IL, U.S.). Each experiment was repeated at least three times. The results were reported as the mean \pm standard deviation (SD). Differences were considered statistically significant at $\mathrm{P}<0.05$.

\section{Results}

\section{GABABR I was down-regulated and negatively associated with the adverse clinicopathological features in colorectal cancer}

To investigate the role of GABABR, we first found that the expression of GABABR1 in tumor tissues was significantly lower than those in normal adjacent tissues from patients with CRC by using immunohistochemistry Compared with the normal adjacent tissues, the rate of GABABR1 positive cells $(5 \%)$ was decreased in tumor tissues (Figure 1A). However, the expression of GABABR2 was too low to detective in both tumor tissues and normal adjacent tissues (Figure 1B). Then, we evaluated the mRNA level of GABABR1 and GABABR2 in ten paired normal adjacent tissues and colon tissues. It was found that the mRNA level of GABABR1 in colon tissues was also significantly decreased (Figure 1C). We next confirmed the decreased protein expression of GABABR1 in colon tissues by Western Blot (Figure 1D. Furthermore, we searched PROGgeneV2 and found two datasets (GSE39582 [19] and GSE41258 [20]). We found that the high expression of GABABR1 was associated with a significant lengthening of survival in CRC patients (Figure 1E and 1F). Then, we used 40 cases of CRC specimens surgically removed during January 2015 to June 2015 at Fudan University Shanghai Cancer Center (FUSCC), and analyzed the effect of GABABR1 expression on overall survival (OS). Patients with higher GABABR1 mRNA level than the average for all patients were assigned to GABABR1 high group and patients with lower GABABR1 mRNA level were assigned to GABABR1 low group. The Kaplan-Meier survival curves showed 
that patients with higher GABABR1 expression also had a significantly better 3-year OS (Figure 1G).

\section{Down-regulation of GABABRI promotes proliferation, migration, and invasion in CRC cells}

Based on the results observed in CRC patients, we detected the protein and mRNA expression of GABABR1 in several human CRC cell lines and finally chose two cell lines (RKO and Lovo) with higher expression of GABABR1as experimental models (Figure 2A and 2B). The GABABR1 level was knockdown by specific shRNA target to GABABR1 mRNA CDS region. The efficiency of knockdown was evaluated by real-time PCR (Figure 2C) and Western Blot (Figure 2D). The results showed that GABABR1 knockdown dramatically improved the proliferation (Figure 2E), migration (Figure 2F), and invasion (Figure 2G) abilities, indicated by CCK-8, wound healing and Matrigel invasion assays, respectively. Meanwhile, we found that the migration rate was significantly reduced in RKO and Lovo cells treated with baclofen (an agonist of GABABR, $10 \mu \mathrm{M}$ )
(Supplementary Figure 1A). To evaluate the action of GABABR on cell invasion ability, we performed BD Matrigel invasion assays with RKO and Lovo cells and found that activation of GABABR reduced the number of invading cells in both cell types (Supplementary Figure 1B). We also assessed the effect of GABABR on the proliferation of CRC cells. We found that activation of GABABR inhibited the proliferation of RKO and Lovo cells (Supplementary Figure 1C). These results demonstrated that down-regulation of GABABR promotes proliferation, migration, and invasion in CRC cells. On the other hand, CGP52432, one of the antagonists of GABABR1, consistently enhanced the proliferation (Supplementary Figure 2A), migration (Supplementary Figure 2B), and invasion (Supplementary Figure 2C) abilities, indicated by CCK-8, wound healing and Matrigel invasion assays, respectively. These results suggested that downregulation of GABABR promotes proliferation, migration, and invasion in CRC cells. a
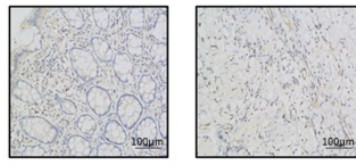

CRC-NT(200x) CRC-T(200x) b

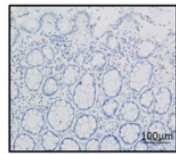

CRC-NT(200x)

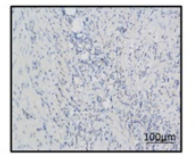

CRC-T $(200 \times)$
C

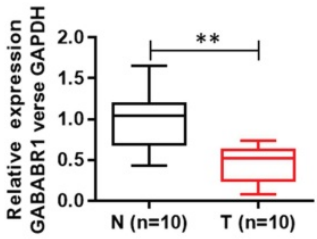

d

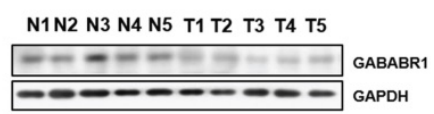

e

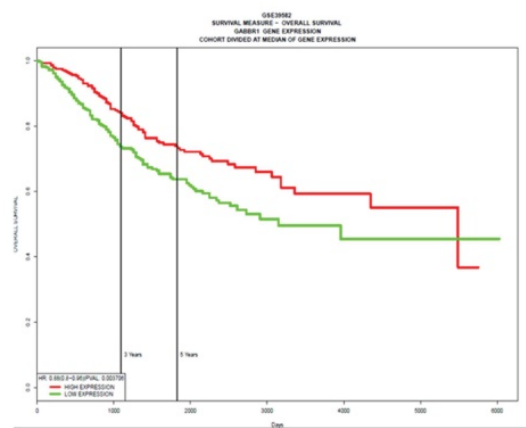

GSE39582 f

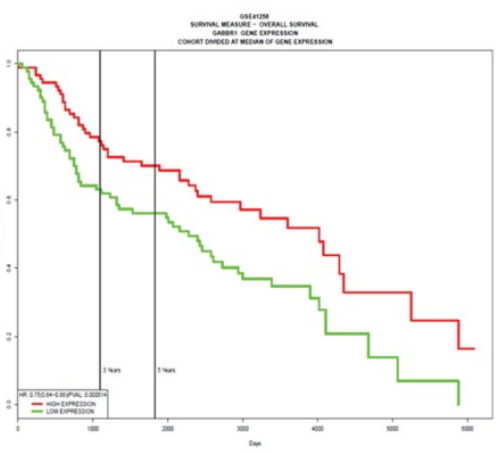

GSE41258

g

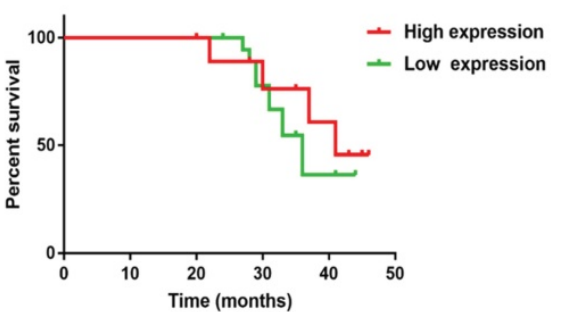

Figure 1. GABABRI was down-regulated and negatively associated with the adverse clinicopathological features in colorectal cancer. (A-B) Representative immunohistochemical staining of GABABR1 and GABABR2 in human CRC tissue (CRC-T) and normal tissues (CRC-NT); scale bar: $100 \mu M$. (C) The mRNA level of GABABR1 in normal adjacent tissues and colon tissues were evaluated by real-time PCR. (D) The protein level of GABABR1 in normal adjacent tissues and colon tissues were evaluated by Western Blot. (E-F) We searched PROGgeneV2 and analyzed the effect of GABABR1 expression on survival of CRC patients. (G) A Kaplan-Meier curve of overall survival in 40 patients is shown with $C R C$ stratified by the expression level of GABABR. The duration of survival was measured from postoperative day 1 until death or 44 months later. $* P<0.05$ versus indicated group. 
a

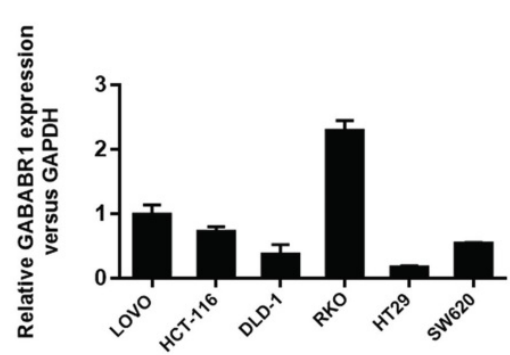

b

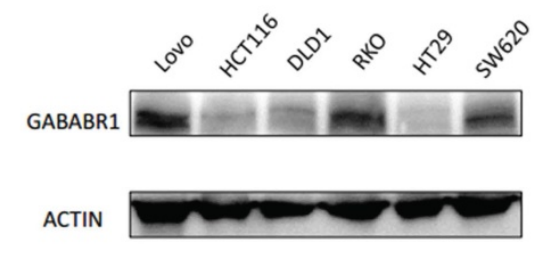

e

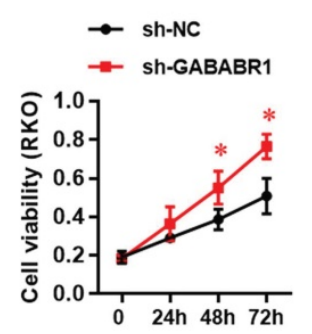

C

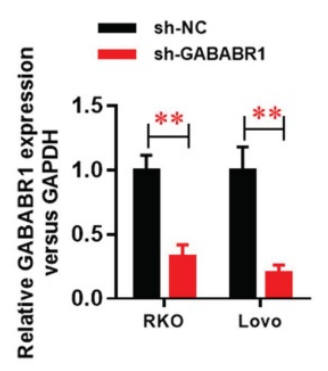

d

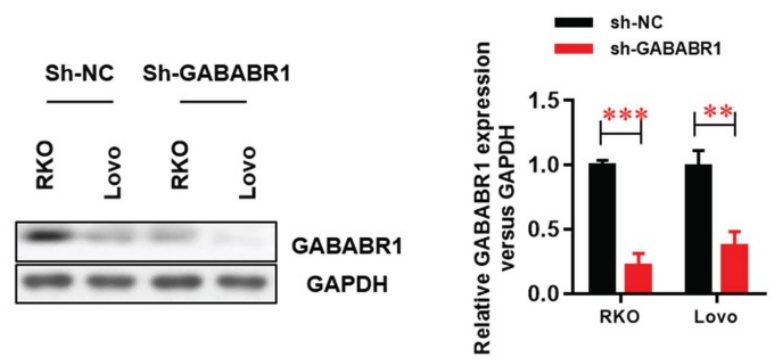

f

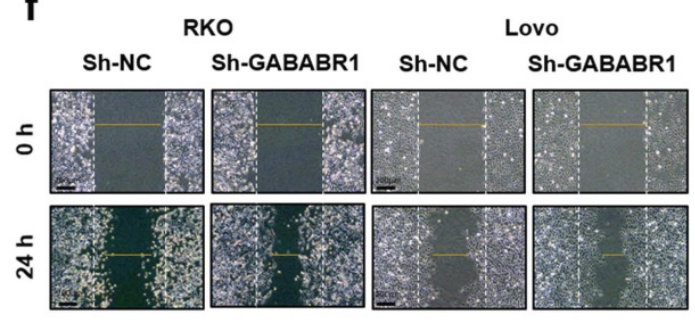

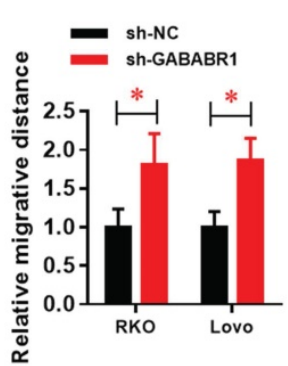

g

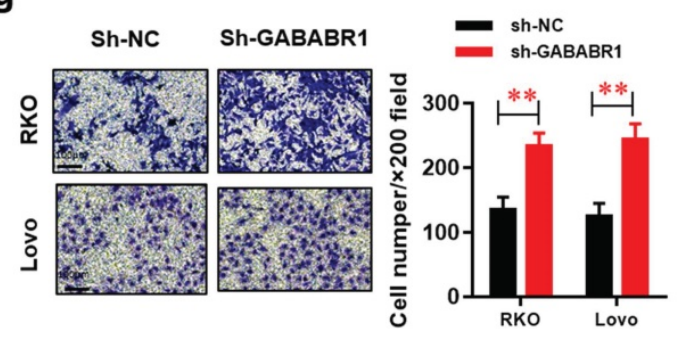

Figure 2. Down-regulation of GABABR promotes proliferation, migration, and invasion in CRC cells. (A) Real-time PCR was used to measure the levels of GABABR in six CRC cell lines. (B) GABABR protein levels were tested by western blot. (C)The efficiency of GABABR1 mRNA knockdown was evaluated by real-time PCR. (D) The efficiency of GABABRI protein knockdown was evaluated by Western Blot. (E) The effect of GABABRI knockdown on the cell viability of RKO and Lovo cells were evaluated by CCK-8 assay. (F) The effect of GABABR1 knockdown on the migration of RKO and Lovo cells were evaluated by wound healing assay. (G) The effect of GABABR1 knockdown on the invasion of RKO and Lovo cells were evaluated by Matrigel invasion assay. $N=3, * P<0.05$ versus indicated group.

\section{Down-regulation of GABABR1 activates EMT in CRC cells}

EMT plays a critical role in tumor migration and invasion [21]. In this study, the protein expression levels of the EMT-related genes $\mathrm{N}$-cadherin and Vimentin were evaluated by Western Blot and found to be elevated, whereas the protein level of E-cadherin was decreased by GABABR1 knockdown (Figure 3A). Moreover, the EMT morphology changes were confirmed, indicating by dysregulated expression of E-cadherin and vimentin were visualized by immunostaining and confocal imaging (Figure 3B).

\section{Activation of Hippo/YAP I signaling contributes to the GABABR I down-regulation promoted proliferation, migration, and invasion in CRC cells}

Studies suggest that the Hippo/YAP pathway is involved in EMT [22]. The datasets from PROGgeneV2 prompt that GABABR may be involved in the regulation of Hippo/YAP pathway. Herein, we found that protein level of functional type YAP1 was elevated in the nucleus fraction of GABABR1 knockdown RKO and Lovo cells (Figure 4A). Meanwhile, the non-functional type YAP1 (phosphorylated YAP1) and its upstream phosphorylated protein LATS1 was decreased. Consistently, the luciferase reporter assay showed that the YAP1-TEAD activity was significantly elevated by GABABR1 knockdown in RKO and Lovo cells (Figure 4B). Interestingly, Peptide 17, a specific inhibitor of YAP-TEAD, made an almost complete reverse on the GABABR1 knockdown elevated proliferation (Figure 4C), migration (Figure 4D), and invasion (Figure 4E) abilities, indicated by CCK-8, wound healing and Matrigel invasion assays, respectively.

\section{Activation of Hippo/YAP I signaling contributes to the GABABRI down-regulation activated EMT in CRC cells}

To further explore whether Activation of Hippo/YAP1 signaling contributes to the GABABR1 down-regulation activated EMT in CRC cells, we then evaluated the mRNAs (Figure $5 \mathbf{A}$ ) and protein 
(Figure 5B) levels of EMT-related genes, including E-cadherin, N-cadherin, and Vimentin. The results showed that the GABABR1 knockdown decreased E-cadherin and increased $\mathrm{N}$-cadherin and Vimentin were also reversed to comparable level with control group. Furthermore, the EMT morphology changes were also confirmed, indicating by reversed expression of E-cadherin and vimentin were visualized by immunostaining and confocal imaging (Figure 5C).

\section{Activation of Hippo/YAP I signaling contributes to the GABABR1 down-regulation increased tumor growth in vivo}

To further evaluated the role of Hippo/YAP1 signaling in the GABABR1 down-regulation impaired biological phenotype of colon cancer cells in vivo, we constructed a colon cancer Xenograft mice model via subcutaneous injection of normal or GABABR1 knockdown Lovo cells with or without Peptide 17 treatment. Xenograft assays showed that GABABR1 knockdown dramatically increased tumor growth compared with the control group (Figure 6A), while YAP1-TEAD inhibition with Peptide 17 reversed tumor volume (Figure 6B) and weight (Figure 6C) regardless of the GABABR1 knockdown. H\&E staining and IHC staining confirmed the expression levels of GABABR1 and YAP1 in these tumors (Figure 6D). These results collectively demonstrated that activation of Hippo/YAP1 signaling contributes to the GABABR1 down-regulation increased tumor growth in vivo.

\section{Discussion}

CRC has been challenging to treat because it tends to recur frequently. However, the molecular and cellular mechanisms of CRC are not entirely known. GABABR was verified to be associated with the progression of various tumors. This study showed that GABABR1 inhibited the Hippo/YAP1 pathway, suppressed the expression of EMT- related protein, and finally alleviated the migration and invasion of CRC.

As an inhibiting neurotransmitter, previous prospective studies on GABABR mainly involved neurons, neuropathic pain and neuroinflammation [23-25]. The role of GABABR in cancer has received increasing attention in recent years. Studies have shown that GABABR is closely associated with tumor cell apoptosis in cancers such as breast cancer, renal carcinoma, and gastric cancer $[5,26]$. Nevertheless, its exact mechanism has not been illustrated thus far. In this study, we first found that the prolonged OS was associated with higher expression of GABABR1, suggesting that patients with lower expression of GABABR1 may have a poor prognosis.

a
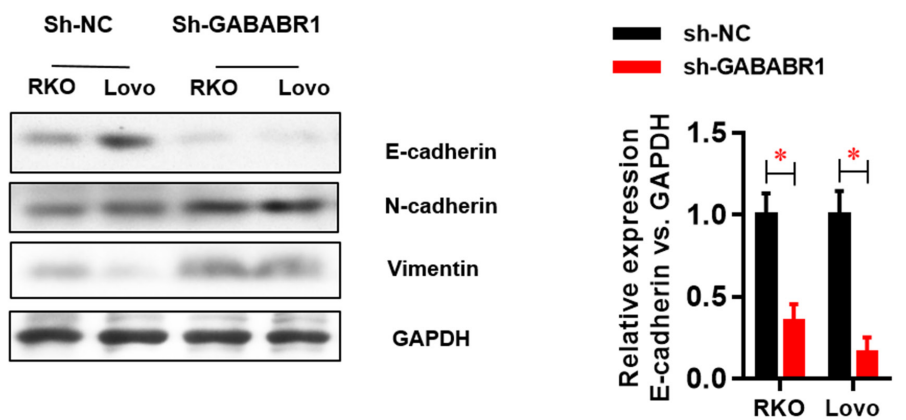
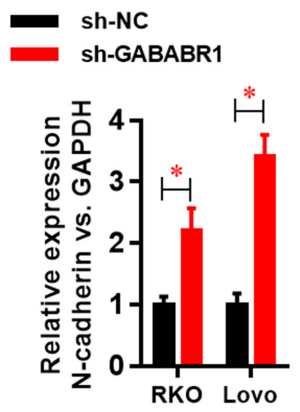
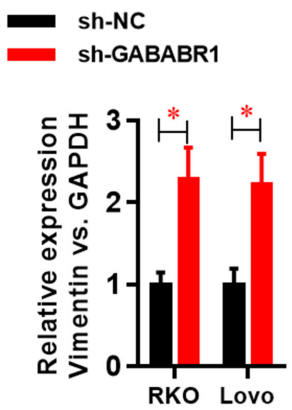

b
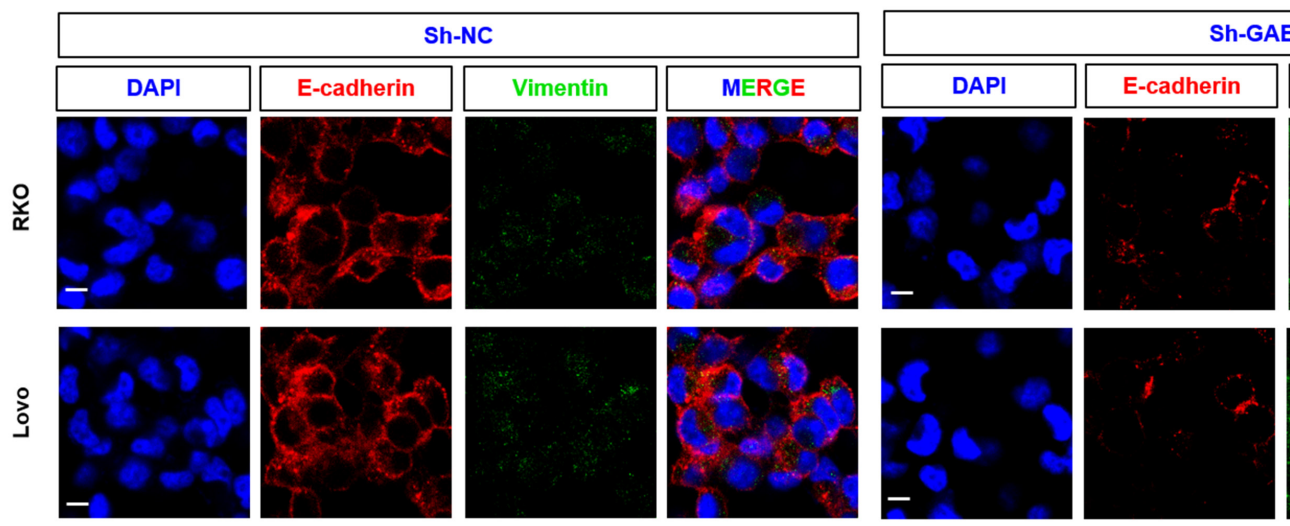

-GABABR1
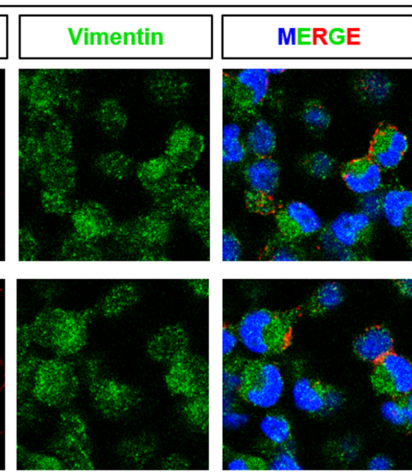

Figure 3. Down-regulation of GABABRI activates EMT in CRC cells. (A) The effect of GABABR1 knockdown on the protein level of EMT related genes, including $\mathrm{E}$-cadherin, $\mathrm{N}$-cadherin and Vimentin were detected by Western Blot. (B) The dysregulated expression of E-cadherin and vimentin were visualized by immunostaining and confocal imaging. $\mathrm{N}=3, * \mathrm{P}<0.05$ versus indicated group. 
a

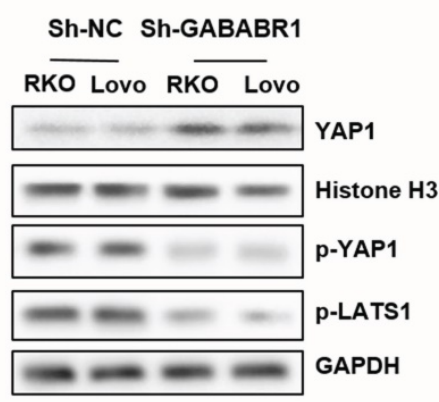

sh-NC

sh-GABABR1

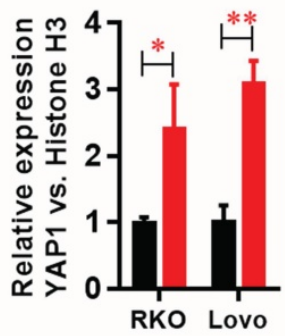

b

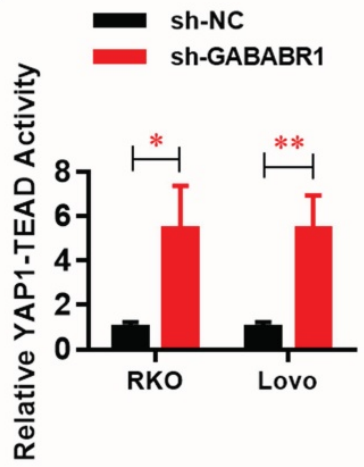

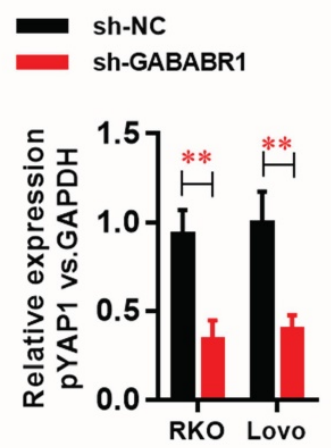

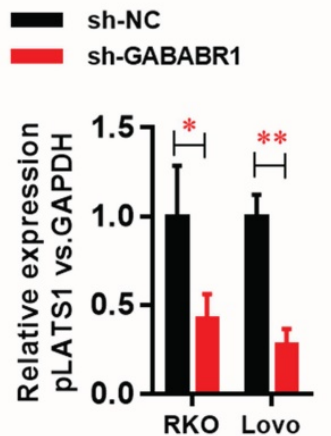

C
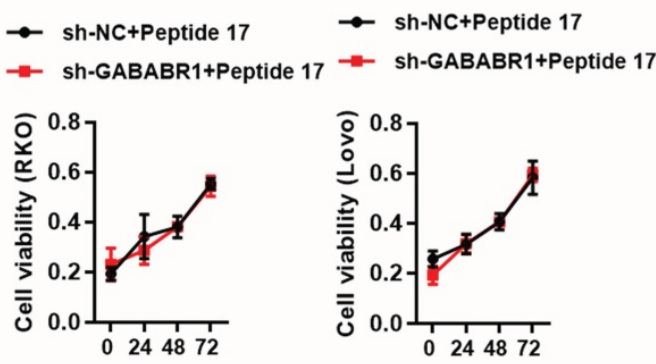

d

RKO+Peptide 17

Lovo+Peptide 17
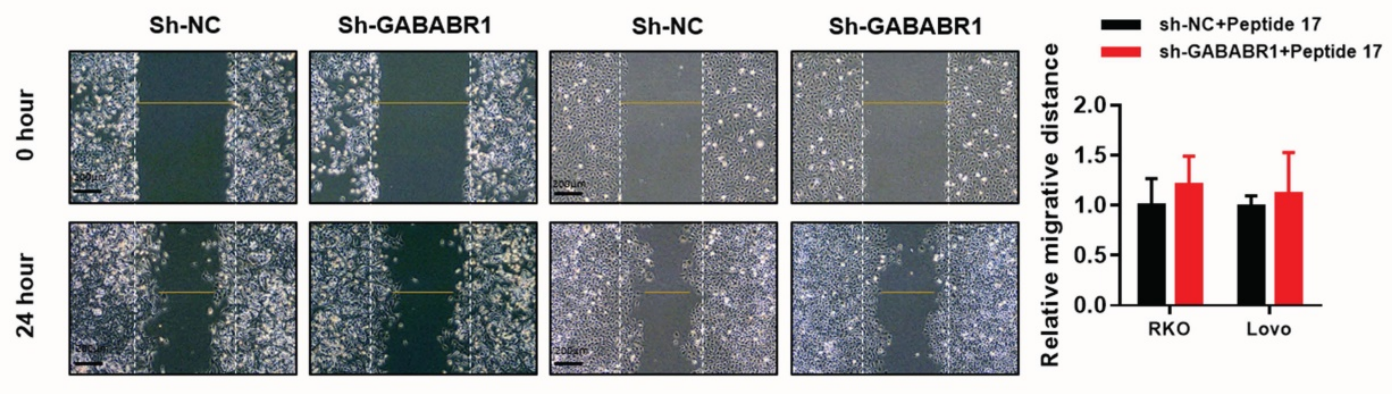

e

RKO+Peptide 17

Lovo+Peptide 17

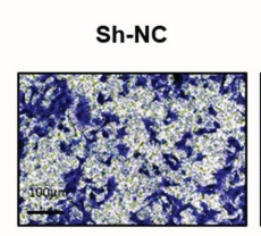

Sh-GABABR1
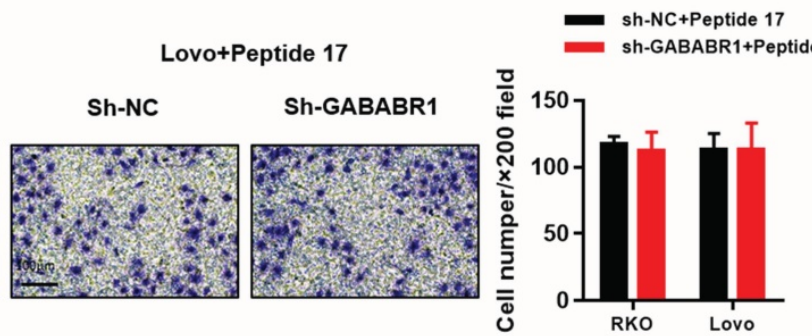

Figure 4. Activation of Hippo/YAPI signaling contributes to the GABABRI down-regulation promoted proliferation, migration, and invasion in CRC cells. (A) The effect of GABABR1 knockdown on the protein level of Hippo-signaling pathway, including nucleus YAPI, phosphorylated YAPI and phosphorylated LATS1 were detected by Western Blot. (B) The effect of GABABR1 knockdown on the activity of Hippo-signaling pathway were detected by luciferase assay. (C) The effect of Peptide 17 (1 $\mu \mathrm{M}$ ) treatment on the cell viability of GABABR1 knockdown RKO and Lovo cells were evaluated by CCK-8 assay. (F) The effect of Peptide 17 treatment on the migration of GABABR 1 knockdown RKO and Lovo cells were evaluated by wound healing assay. (G) The effect of Peptide 17 treatment on the invasion of GABABR1 knockdown RKO and Lovo cells were evaluated by Matrigel invasion assay. $N=3, * P<0.05$ versus indicated group.

Metastatic recurrence is the major obstacle preventing improvement of the prognosis for CRC patients. In investigating the function of GABABR1 in CRC progression, we first found that activation of GABABR1 attenuated the proliferation of CRC cells. This was consistent with previous research by Shu et al. [9]. Then, we tested the migration rate and number of invading cells in RKO and Lovo cells treated with baclofen. Our results showed that activation of GABABR1 alleviated CRC cell migration and invasion. To test this further, we used CGP52432 or shRNA to inhibit the expression of GABABR1. Contrary to previous results, downregulation of GABABR1 promoted CRC cell migration and invasion. These results helped us confirm that GABABR1 inhibits migration and invasion of CRC. 


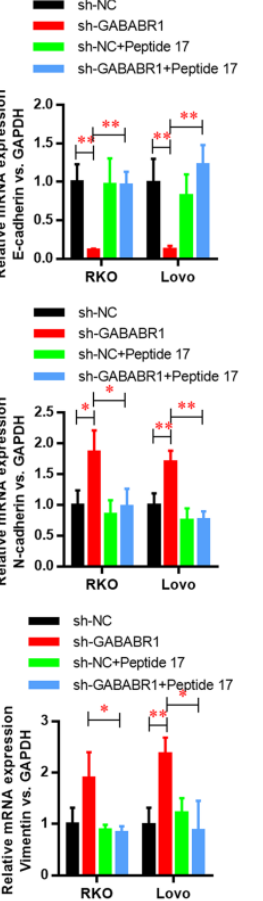

b

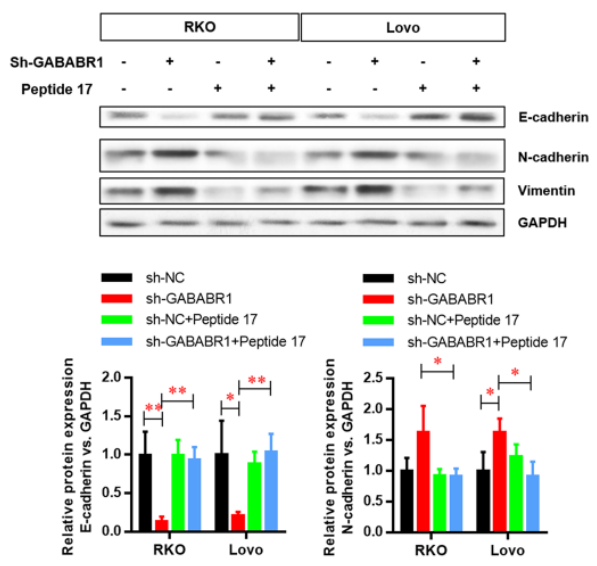

-

- sh-GABABR1 Sh-NCHPaple 17

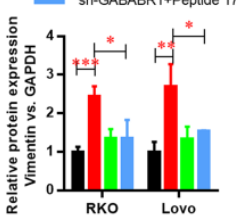

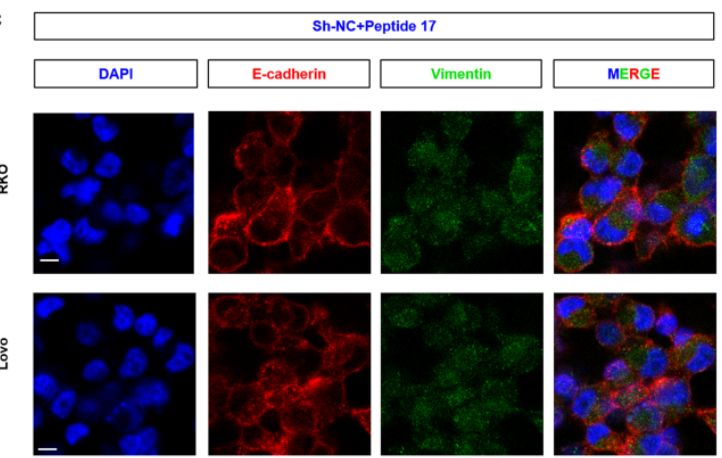
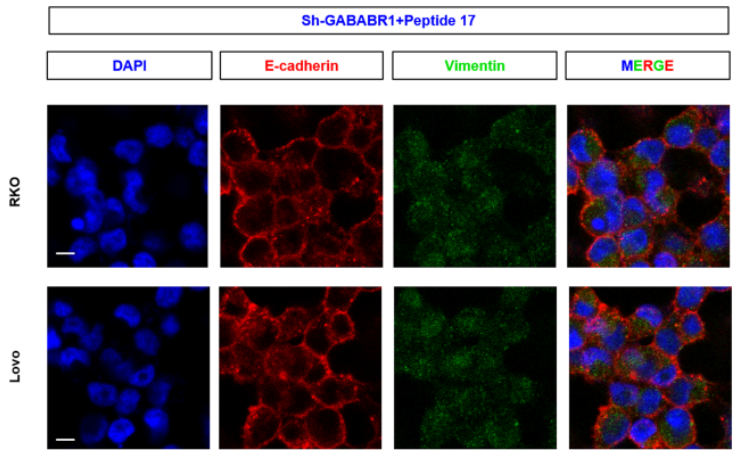

Figure 5. Activation of Hippo/YAPI signaling contributes to the GABABRI down-regulation activated EMT in CRC cells. (A) The effect of Peptide 17 treatment on the mRNA level of EMT related genes, including E-cadherin, N-cadherin and Vimentin were detected by Western Blot. (B) The effect of Peptide 17 treatment on the protein level of EMT related genes, including E-cadherin, $\mathrm{N}$-cadherin and Vimentin were detected by Western Blot. (C) The dysregulated expression of E-cadherin and vimentin were visualized by immunostaining and confocal imaging. $N=3, * P<0.05$ versus indicated group.

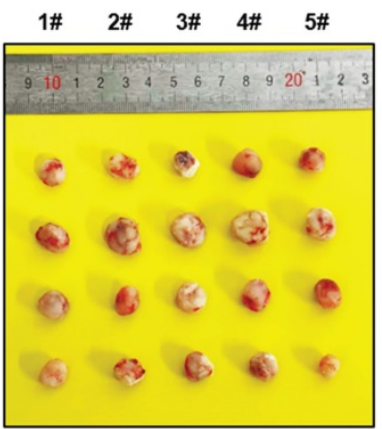

b

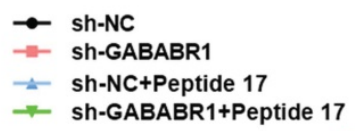

Sh-NC

Sh-GABABR1

Sh-NC+Peptide 17

Sh-GABABR1 + Peptide 17

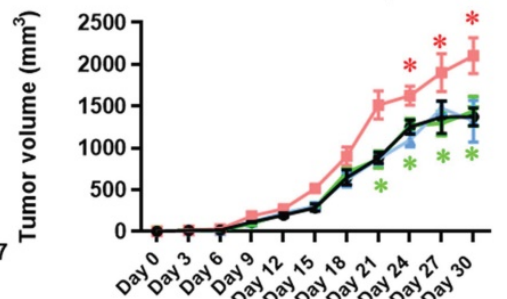

C

- sh-NC

- sh-GABABR1

sh-NC+Peptide 17

sh-GABABR1+Peptide 17

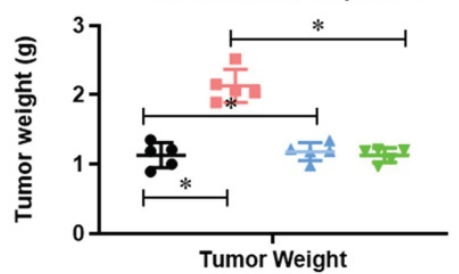

d
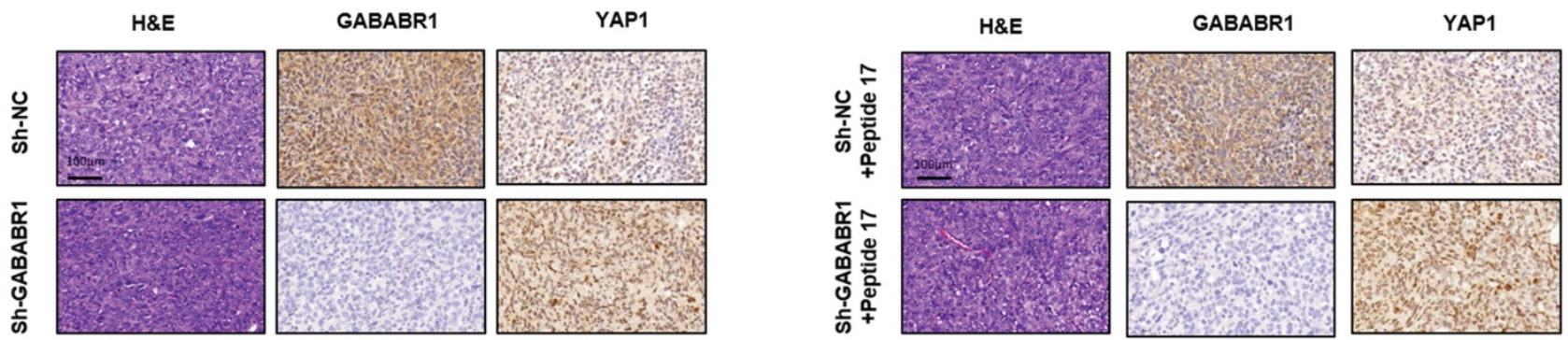

Figure 6. Activation of Hippo/YAPI signaling contributes to the GABABRI down-regulation increased tumor growth in vivo. (A) Representative photographs of the tumor-xenografted nude mice. (B) The tumor growth curve of the nude mice bearing Lovo cells with or without GABABRI knockdown or Peptide 17 treatment. (C) The tumor weight of the nude mice bearing Lovo cells with or without GABABR1 knockdown or Peptide 17 treatment. (D) H\&E, GABABR1 and YAPI staining of subcutaneous tumors derived from Lovo cells with or without GABABR1 knockdown or Peptide 17 treatment. Scale bars: $100 \mu M$. $N=5$, $* P<0.05$ versus indicated group.

To understand how GABABR1 regulates CRC migration and invasion, we further analyzed the datasets from PROGgeneV2 and found that GABABR1 may be involved in the regulation of
Hippo/YAP pathway. It is well known that YAP plays a role in promoting tumorigenesis, cell proliferation, and resistance to apoptosis in tumor cells [10]. Here, we examined YAP1 and the 
phosphorylation of YAP1 and LATS1 and YAP-TEAD activity. We found that downregulation of GABABR1 promoted activation of the Hippo/YAP1 pathway in GABABR1 knockdown RKO and Lovo cells. However, treatment with Peptide 17 made an almost complete reverse on the GABABR1 knockdown elevated proliferation, migration and invasion abilities, respectively. This indicated that GABABR1 probably regulated EMT through Hippo/YAP pathway. Recently, research has indicated that the YAP pathway is involved in the regulation of EMT and metastasis [27, 28]. EMT, an essential regulatory process that mediates invasion and metastasis, is considered a culprit of metastasis in CRC [29]. There are many pathways upstream of EMT, such as Wnt $\beta$-catenin, JNK, and TGF- $\beta[25,26]$. In this study, the expression of EMT-related molecules $\mathrm{N}$-cadherin and Vimentin were found to be elevated, and E-cadherin was suppressed (at the protein and mRNA level) in GABABR1 knockdown RKO and Lovo cells. Then, this trend partly reversed with piptide 17 treatment. Therefore, GABABR1 might inhibit the EMT process to regulate CRC migration and invasion by Hippo/YAP1 pathway. To further investigate the role of GABABR1 and Hippo/YAP pathway, we constructed a colon cancer Xenograft mice model. The results showed that activation of Hippo/YAP1 signaling contributes to the GABABR1 downregulation increased tumor growth in vivo. These results revealed that the Hippo/YAP pathway might be upstream of EMT and that GABABR inhibits migration and invasion via the Hippo/YAP pathway and EMT.

Taken together, these findings indicate that GABABR1 exhibits an anti-tumor effect. The aberrant expression of GABABR1 regulates the EMT to suppress migration and invasion through the Hippo/YAP1 pathway, implicating GABABR1 as a therapeutic target in CRC.

\section{Abbreviations}

GABABR: Gamma-Aminobutyric Acid Type B Receptor; YAP: Yes-associated protein; LATS1: large tumor suppressors 1.

\section{Supplementary Material}

Supplementary figures and tables.

http://www.ijbs.com/v17p1953s1.pdf

\section{Acknowledgements}

\section{Funding}

This research was supported by the National Key Research and Development Program of China (NO. 2020YFC2008400), the National Natural Science
Foundation of China (NO.81873948, 81871591), Clinical Research Plan of SHDC (NO. SHDC2020CR4064, SHDC2020CR1005A), Shanghai Shenkang hospital development center clinical science and technology innovation project (NO. SHDC12018105), the Key Technology and Development Program of Shanghai (NO. 17411963400); 2019 Fudan University Zhuo-Xue Project (JIF159607); Shanghai Leading Talent (NO: 2019-112).

\section{Competing Interests}

The authors have declared that no competing interest exists.

\section{References}

1. Bray F, Ferlay J, Soerjomataram I, Siegel RL, Torre LA, Jemal A. Global cancer statistics 2018: GLOBOCAN estimates of incidence and mortality worldwide for 36 cancers in 185 countries. CA Cancer J Clin. 2018; 68: 394-424.

2. Keum N, Giovannucci E. Global burden of colorectal cancer: emerging trends, risk factors and prevention strategies. Nat Rev Gastroenterol Hepatol. 2019.

3. Frangaj A, Fan QR. Structural biology of GABAB receptor. Neuropharmacology. 2018; 136: 68-79.

4. George K, Sadiq NM. GABA Inhibitors. StatPearls. Treasure Island (FL); 2019.

5. Tian H, Wu JX, Shan FX, Zhang SN, Cheng Q, Zheng JN, et al. Gamma-aminobutyric acid induces tumor cells apoptosis via GABABR1.beta-arrestins.JNKs signaling module. Cell Biochem Biophys. 2015; 71: 679-88.

6. Kanbara K, Otsuki Y, Watanabe M, Yokoe S, Mori Y, Asahi M, et al. GABAB receptor regulates proliferation in the high-grade chondrosarcoma cell line OUMS-27 via apoptotic pathways. BMC Cancer. 2018; 18: 263.

7. Gao J, Lin S, Gao Y, Zou X, Zhu J, Chen M, et al. Pinocembrin inhibits the proliferation and migration and promotes the apoptosis of ovarian cancer cells through down-regulating the mRNA levels of $\mathrm{N}$-cadherin and GABAB receptor. Biomed Pharmacother. 2019; 120: 109505.

8. Solorzano SR, Imaz-Rosshandler I, Camacho-Arroyo I, Garcia-Tobilla P, Morales-Montor G, Salazar P, et al. GABA promotes gastrin-releasing peptide secretion in NE/NE-like cells: Contribution to prostate cancer progression. Sci Rep. 2018; 8: 10272.

9. Shu Q, Liu J, Liu $X$, Zhao $\mathrm{S}$, Li $\mathrm{H}$, Tan $\mathrm{Y}$, et al. GABAB R/GSK-3beta/NF-kappaB signaling pathway regulates the proliferation of colorectal cancer cells. Cancer Med. 2016; 5: 1259-67.

10. Kim CL, Choi SH, Mo JS. Role of the Hippo Pathway in Fibrosis and Cancer. Cells. 2019; 8

11. Azad T, Nouri K, Janse van Rensburg HJ, Maritan SM, Wu L, Hao Y, et al. A gain-of-functional screen identifies the Hippo pathway as a central mediator of receptor tyrosine kinases during tumorigenesis. Oncogene. 2019.

12. Chen W, Wang H, Liu Y, Xu W, Ling C, Li Y, et al. Linc-RoR promotes proliferation, migration, and invasion via the Hippo/YAP pathway in pancreatic cancer cells. J Cell Biochem. 2020; 121: 632-41.

13. Zhou H, Li G, Huang S, Feng Y, Zhou A. SOX9 promotes epithelial-mesenchymal transition via the Hippo-YAP signaling pathway in gastric carcinoma cells. Oncol Lett. 2019; 18: 599-608.

14. Mao J, Tian Y, Wang C, Jiang K, Li R, Yao Y, et al. CBX2 Regulates Proliferation and Apoptosis via the Phosphorylation of YAP in Hepatocellular Carcinoma. J Cancer. 2019; 10: 2706-19.

15. Zhou L, Cai L, Guo Y, Zhang H, Wang P, Yi G, et al. Calotropin activates YAP through downregulation of LATS1 in colorectal cancer cells. Onco Targets Ther. 2019; 12: 4047-54.

16. Hao F, Xu O, Zhao Y, Stevens JV, Young SH, Sinnett-Smith J, et al. Insulin Receptor and GPCR Crosstalk Stimulates YAP via PI3K and PKD in Pancreatic Cancer Cells. Mol Cancer Res. 2017; 15: 929-41.

17. Yu FX, Zhao B, Panupinthu N, Jewell JL, Lian I, Wang LH, et al. Regulation of the Hippo-YAP pathway by G-protein-coupled receptor signaling. Cell. 2012; 150: 780-91.

18. Luo J, Yu FX. GPCR-Hippo Signaling in Cancer. Cells. 2019; 8.

19. Marisa L, de Reynies A, Duval A, Selves J, Gaub MP, Vescovo L, et al. Gene expression classification of colon cancer into molecular subtypes: characterization, validation, and prognostic value. PLoS Med. 2013; 10: e1001453.

20. Sheffer M, Bacolod MD, Zuk O, Giardina SF, Pincas H, Barany F, et al Association of survival and disease progression with chromosomal instability: a genomic exploration of colorectal cancer. Proc Natl Acad Sci U S A. 2009; 106: 7131-6.

21. Jolly MK, Celia-Terrassa T. Dynamics of Phenotypic Heterogeneity Associated with EMT and Stemness during Cancer Progression. J Clin Med. 2019; 8. 
22. Santoro R, Zanotto M, Carbone C, Piro G, Tortora G, Melisi D. MEKK3 Sustains EMT and Stemness in Pancreatic Cancer by Regulating YAP and TAZ Transcriptional Activity. Anticancer Res. 2018; 38: 1937-46.

23. Zhu YS, Xiong YF, Luo FQ, Min J. Dexmedetomidine protects rats from postoperative cognitive dysfunction via regulating the GABAB R-mediated cAMP-PKA-CREB signaling pathway. Neuropathology. 2019; 39: 30-8.

24. Li Z, Liu P, Zhang H, Zhao S, Jin Z, Li R, et al. Role of GABAB receptors and p38MAPK/NF-kappaB pathway in paclitaxel-induced apoptosis of hippocampal neurons. Pharm Biol. 2017; 55: 2188-95.

25. Winkler M, Biswas S, Berger SM, Kuchler M, Preisendorfer L, Choo M, et al. Pianp deficiency links GABAB receptor signaling and hippocampal and cerebellar neuronal cell composition to autism-like behavior. Mol Psychiatry. 2019

26. Zhang D, Li X, Yao Z, Wei C, Ning N, Li J. GABAergic signaling facilitates breast cancer metastasis by promoting ERK1/2-dependent phosphorylation. Cancer Lett. 2014; 348: 100-8.

27. Dai BW, Yang ZM, Deng P, Chen YR, He ZJ, Yang X, et al. HOXC10 promotes migration and invasion via the WNT-EMT signaling pathway in oral squamous cell carcinoma. J Cancer. 2019; 10: 4540-51.

28. Zheng X, Chen L, Zhou Y, Wang Q, Zheng Z, Xu B, et al. A novel protein encoded by a circular RNA circPPP1R12A promotes tumor pathogenesis and metastasis of colon cancer via Hippo-YAP signaling. Mol Cancer. 2019; 18: 47.

29. Vu T, Datta PK. Regulation of EMT in Colorectal Cancer: A Culprit in Metastasis. Cancers (Basel). 2017; 9 . 